\title{
Prevalence and Antibiotic Resistance Pattern of Salmonella typhi and Salmonella paratyphi A isolated by Automated Blood Culture System
}

\author{
Md. Badrul Islam ${ }^{1}$, Sazzad Bin Shahid ${ }^{2}$, AFM Arshedi Satar ${ }^{3}$, Md. Abdullah Yusuf $^{4}$, Shoriful \\ Islam $^{5}$, Raihanul Islam ${ }^{6}$
}

\begin{abstract}
${ }^{1}$ Associate Professor, Department of Microbiology, Dhaka National Medical College, Dhaka, Bangladesh; ${ }^{2}$ Associate Professor, Department of Microbiology, Dhaka Medical College, Dhaka, Bangladesh; ${ }^{3}$ Associate Professor, Department of Microbiology, National Institute of Neurosciences \& Hospital, Dhaka, Bangladesh; ${ }^{4}$ Assistant Professor, Department of Microbiology, National Institute of Neurosciences \& Hospital, Dhaka, Bangladesh; ${ }^{5}$ Medical Technologist (Lab), IBN Sina Diagnostic Lab and Consultation center, Doyagonj, Dhaka, Bangladesh; 'Lab Biochemist, IBN Sina Diagnostic Lab and consultation Center, Doyagonj, Dhaka, Bangladesh
\end{abstract}

[Received: 12 July 2020; Accepted: 30 August 2020; Published: 1 December 2020]

\begin{abstract}
Background: Salmonella thyphi and Salmonella paratyphi A are the members of Enterobactoriaceae and gram negative rods causing typhoid fever \& partyphoid fever respectively. Automated blood culture system is the standard diagnostic method. Objective: The purpose of the present study was to see the prevalence and antibiotic resistance pattern of Salmonella typhi and Salmonella paratyphi A isolated by automated blood culture system. Methodology: This cross-sectional study was done in the Microbiology Lab at IBN Sina D. Lab and consultation center, Doyagonj, Dhaka, Bangladesh from January 2019 to June 2019. Blood culture was performed by automated blood culture method. Sensitivity pattern of antibiotic was measured by Disk diffusion method. Result: A total of 3240 blood samples were collected from suspected patients. Among them, bacteria were isolated 336 (10.37\%). The most common isolated bacteria were Salmonella typhi which was 261(77.68\%) cases and Salmonella paratyphi A which was in $60(17.86 \%)$ cases. Salmonella typhi is the most common organism and showed sensitivity pattern to imipenem $97.3 \%$, colistin 80.46\% and amikain $77.4 \%$ and Salmonella paratyphi A showed. Sensitivity pattern to imipenem $93.3 \%$ and amikacin 83.3\%. Conclusion: In conclusion high rate of Salmonella typhi and Salmonella paratyphi A are isolated during blood culture showing less sensitive to imipenem and amikacin. [Bangladesh Journal of Infectious Diseases, December 2020;7(2):57-60]
\end{abstract}

Keywords: Prevalence; Antibiotic Resistance Pattern; Salmonella typhi; Salmonella paratyphi A; Automated Blood Culture System

Correspondence: Dr. Md. Badrul Islam, Associate Professor, Department of Microbiology, Dhaka National Medical College, Dhaka, Bangladesh; Email: badrulislam19@gmail.com; Cell no.: +8801670738692

Conflict of interest: Authors declare that there is no conflict of interests.

Funding agency: The study was not funded by any authority.

Contribution to authors: Islam MB, Shahid SB involved in protocol preparation, data collection and literature search up to manuscript writing. Lab work and data were collected by Islam S, Islam R. Satar AFMA, Yusuf MA involved in literature search, preparation and revision of this manuscript.

How to cite this article: Islam MB, Shahid SB, Satar AFMA, Yusuf MA, Islam S, Islam R. Prevalence and Antibiotic Resistance Pattern of Salmonella typhi and Salmonella paratyphi A isolated by Automated Blood Culture System. Bangladesh J Infect Dis 2020;7(2):57-60

Copyright: (02020. Islam et al. Published by Bangladesh Journal of Infectious Diseases. This article is published under the Creative Commons CC BY-NC License (https://creativecommons.org/licenses/by-nc/4.0/). This license permits use, distribution and reproduction in any medium, provided the original work is properly cited, and is not used for commercial purposes. 


\section{Introduction}

Enteric fever is endemic in Bangladesh with Salmonella enterica var Typhi (S. Typhi) and Salmonella enterica var Paratyphi A (S. Paratyphi A) being the major causative agents ${ }^{1}$. These human restricted pathogens are transmitted by the faeco-oral route in regions with poor standards of hygiene and sanitation accounting for high morbidity and mortality ${ }^{2}$. Antibiotic therapy constitutes the mainstay of management of enteric fever; mortality being as high as $30 \%$ in untreated cases, which falls to less than $1 \%$ with appropriate antibiotic therapy ${ }^{3}$. Failure to treat an infection properly leads to prolonged illness, thus increasing the chance of developing a carrier state in which persons are contagious and able to spread the resistant strain to others. In the last few decades, the emergence of multidrug resistant (MDR) salmonellae (resistant to ampicillin, chloramphenicol and co-trimoxazole) has led to widespread use of fluoroquinolones and thirdgeneration cephalosporins as the first-line drugs ${ }^{1-2}$.

Currently, changes in the epidemiology and drug resistance profile of enteric fever have been noted by various workers. Firstly, many of the researchers have reported an increasing trend of $S$. Paratyphi A over the last decade 4 . Secondly, re-emergence of susceptibility to conventional first-line antibiotics (ampicillin, co-trimoxazole and chloramphenicol) and emergence of reduced susceptibility towards ciprofloxacin among salmonellae have been reported $^{5}$. Furthermore, $S$. Typhi resistant to thirdgeneration cephalosporins, though low at present $(1 \%)$ is also emerging in India ${ }^{6}$.

Various methods are available for blood culture for isolation of salmonella typhi and Salmonella paratyphi A such as conventional methods, semiautomated methods and automated methods ${ }^{7}$. Automated method is the best of them, like Bac T/Alert. Conventional blood culture methods often yield poor results because of low bacterial load and increased chance of contamination. The purpose of the present study was to see the prevalence and antibiotic resistance pattern of Salmonella typhi and Salmonella paratyphi A isolated by automated blood culture system.

\section{Methodology}

The cross sectional study was done in IBN Sina D. Lab \& consultation center, Doyagonj, Dhaka, Bangladesh from January 2019 to June 2019. A total of 3240 blood samples were collected aseptically from different sexes and different age groups. All blood samples were placed in blood culture matching Positive blood culture samples were inoculated in blood agar, chocolate agar and MacConkey's agar media. All plates were incubated at 370C aerobically for 24 hours. After incubation, plates were checked for presence of suspected organisms. All the microorganisms were identified by their colony morphology, staining character, motility, pigment production, catalase, oxidase, TSI, MIU citrate tests ${ }^{1}$. Isolated bacteria were tested for antimicrobial susceptibility Muller Hinton agar media against different antimicrobial agents ${ }^{2}$. Date were analyzed by statistical package for social science (SPSS).

\section{Results}

A total of 3240 blood samples were collected from suspected patients. From the 3240 blood samples, bacteria were isolated $336(10.37 \%)$.

Table 1: Distribution of Samples of the Study

\begin{tabular}{|l|c|c|}
\hline Culture & Frequency & Percent \\
\hline Positive & 336 & 10.4 \\
\hline Negative & 2904 & 89.6 \\
\hline Total & $\mathbf{3 2 4 0}$ & $\mathbf{1 0 0 . 0}$ \\
\hline
\end{tabular}

Salmonella typhi was isolated 261(77.68\%) cases and Salmonella paratyphi A was in 60(17.86\%) cases (Table 2).

Table 2: Distribution of Isolated Bacteria in blood Samples $(\mathbf{n}=336)$

\begin{tabular}{|l|c|c|}
\hline Isolated Bacteria & Frequency & Percent \\
\hline Salmonella typhi & 261 & 77.68 \\
\hline Salmonella paratyphi A & 60 & 17.86 \\
\hline Escherichia coli & 5 & 1.49 \\
\hline Staph. aures & 5 & 1.49 \\
\hline Klebsiella species & 3 & 0.89 \\
\hline Pseudomonas species & 2 & 0.60 \\
\hline Total & $\mathbf{3 3 6}$ & $\mathbf{1 0 0 . 0}$ \\
\hline
\end{tabular}

Salmonella typhi showed high degrees of sensitivity to imipenem (97.32\%). colistin $(80.46 \%)$ and amikacin (77.40\%). On the other hand, Salmonella paratyphi A showed high degree of sensitivity to imipenem $(93.33 \%)$ colistin $(83.33 \%)$ and amikacin $(83.33 \%)$. However, the low level of sensitivity was found in cephradine (27.2\%), nalidixic acid $(13.41 \%)$ and ciprofloxacin $(50.58 \%)$ towards isolates of Salmonella typhi. In contrast Salmonella paratyphi A showed similar sensitivity pattern (Table 3). 
Table 3: Sensitivity Pattern of Salmonella typhi and Salmonella paratyphi $A$ to different antimicrobial drugs

\begin{tabular}{|l|c|c|}
\hline \multirow{2}{*}{$\begin{array}{l}\text { Antimicrobial } \\
\text { Drugs }\end{array}$} & $\begin{array}{c}\text { Salmonella } \\
\text { typhi } \\
\text { (n=261) }\end{array}$ & $\begin{array}{c}\text { Salmonella } \\
\text { paratyphi A } \\
\text { (n=60) }\end{array}$ \\
\cline { 2 - 3 } Sensitive & Sensitive \\
\hline Amikacin & $77.40 \%$ & $83.33 \%$ \\
\hline Azithromycin & $45.98 \%$ & $25.0 \%$ \\
\hline Ceftazidime & $63.6 \%$ & $41.67 \%$ \\
\hline Ceftriaxone & $91.95 \%$ & $63.33 \%$ \\
\hline Cefixime & $72.8 \%$ & $43.33 \%$ \\
\hline Cefuroxime & $68.97 \%$ & $26.67 \%$ \\
\hline Cephradine & $27.2 \%$ & $20.0 \%$ \\
\hline Ciprofloxacin & $50.58 \%$ & $46.67 \%$ \\
\hline Colistin & $80.46 \%$ & $83.33 \%$ \\
\hline Cotrimoxazole & $49.81 \%$ & $41.67 \%$ \\
\hline Gentamycin & $65.13 \%$ & $63.3 \%$ \\
\hline Nalidixic acid & $13.41 \%$ & $25.00 \%$ \\
\hline Imipenem & $97.32 \%$ & $93.33 \%$ \\
\hline $\begin{array}{l}\text { Tazobactam } \\
\text { Piperacillin }\end{array}$ & $80.84 \%$ & $53.33 \%$ \\
\hline $\begin{array}{l}\text { Amoxycillin } \\
\text { Clavulanic } \\
\text { acid }\end{array}$ & $81.22 \%$ & $75.00 \%$ \\
\hline
\end{tabular}

\section{Discussion}

Automated blood culture system mechanical system to incubate, agitate or monitor blood culture bottles for microbial growth. Blood culture is the standard diagnostic method, provided a large volume of blood is cultured (10 to $20 \mathrm{ml}$ ), they are positive in $60.0 \%$ to $80.0 \%$ of patients with typhoid ${ }^{7}$. Salmonella typhi and Salmonella paratyphi A are diagnosed by using combination of clinical presentation, isolation of the both organisms from body fluid (Blood). The rate of isolation of these bacteria from blood $90 \%$ in untreated patients in the first week ${ }^{8}$.

The sensitivity of blood culture is higher in the first week of the illness which is reduced by prior use of antibiotics ${ }^{9}$. Multiple positive blood culture results are $73.0 \%$ to $97.0 \%$ specific for salmonella typhi ${ }^{6}$. In this study, Salmonella typhi and Salmonella paratyphi A were isolated, 261(77.68\%) and $60(17.86 \%)$ respectively. The microorganisms isolated in the present study are similar to those reported $^{2,6,10}$. Earlier studies have shown that a single mutation in chromosomal gyr A gene (encoding for subunit of DNA gyrase) located in the quinolone resistance determining region (QRDR) are found in most of such isolates, whereas multiple mutations in QRDR confer high level of resistance to fluoroquinolone (MIC ciprofloxacin $\geq 4.0 \mu \mathrm{g} / \mathrm{mL}$ ) and to NA. Plasmid mediated quinolone resistance and chromosomal gyr B mutation results in isolates with DCS and modest elevation of NA MIC ${ }^{11}$. Although we did not determine the molecular mechanism of quinolone resistance in the present study but, from MIC values for ciprofloxacin and NA, it appears that these isolates may be harbouring the chromosomal mutation in gyr A gene (DCS with NA resistance) in $84.5 \%$ of isolates and multiple mutations (chromosomal gyr A and gyr B gene with ciprofloxacin MIC $\geq 4 \mu \mathrm{g} / \mathrm{mL}$ and NA resistance) in $13.8 \%$ of isolates. Decreasing susceptibility of $S$. Typhi to ciprofloxacin has been well documented in several studies and is indicative of the effects of indiscriminate use of this group of antibiotics ${ }^{12}$.

$S$. Typhi resistant to third generation cephalosporins have been long reported from our neighbouring countries and though very low at present (1\%), such strains are also emerging in India ${ }^{11}$. However, we found no resistance during the study period based on disk diffusion testing and $E$-test. This emphasizes the importance of this group of antibiotic as a reserve drug for treating MDR and ciprofloxacin resistant cases.

Trials have demonstrated that azithromycin compares favourably with ceftriaxone in terms of clinical and microbiological cure rates with ease of administration and lower relapse rate for the treatment of uncomplicated typhoid fever ${ }^{4-5}$. Studies on human volunteers have shown that neutrophil concentrations of azithromycin are 100 times more than the serum concentration with a long half-life (23 days). The efficacy of azithromycin during treatment is related to this tissue concentration rather than the serum concentration. This however warrants further trials to know the exact role of azithromycin, which is an orally effective drug in the endemic areas in view of non-uniform susceptibility pattern noticed in various studies.

\section{Conclusion}

In conclusion high rate of isolation of Salmonella typhi is isolated form the blood. Several methods can be performed to isolate the microorganism from blood culture. The antibiotic sensitivity pattern shows a low sensitivity towards the previously used common antibiotics. A large scale study should be conducted to see the real scenario.

\section{References}

1. Cheesbrough M. District laboratory practice in tropical countries. ELBS, Cambridgeshire. England. 2000;2:175-80 
2. Jain S, Chugh TD. Antimicrobial resistance among blood culture isolates of Salmonella enterica in New Delhi. J Infect Developing Countries 2013;7(11):788-95

3. Parry CM, Hein TT, Dougan G, White J Med 2002;347(22):1770-1782

4. Gandra S, Mojica N, Klein EY, Ashok A, Nerurkar V, Kumari M, et al. Trends in antibiotic resistance among major bacterial pathogens isolated from blood cultures tested at a large private laboratory network in India, 2008-2014. International Journal of Infectious Diseases. 2016;50:75-82

5. Singhal L, Gupta PK, Kale P, Gautam V, Ray P. Trends in antimicrobial susceptibility of Salmonella Typhi from North India (2001-2012). Indian journal of medical microbiology. 2014;32(2):149

6. Iyer RN, Jangam RR, Jacinth A, Venkatalakshmi A, Nahdi FB. Prevalence and trends in the antimicrobial susceptibility pattern of Salmonella enterica serovars Typhi and paratyphi A among children in a pediatric tertiary care hospital in South India over a period of ten years: a retrospective study. European J Clinical Microbiol Infect Dis 2017;36(12):2399-404

7. Zellweger RM, Basnyat B, Shrestha P, Prajapati KG, Dongol S, Sharma PK, et al. Thompson CN, Thwaites GE. A 23-year retrospective investigation of Salmonella Typhi and Salmonella
Paratyphi isolated in a tertiary Kathmandu hospital. PLoS neglected tropical diseases. 2017;11(11):e0006051

8. Porwal A, Bhat S. Antibiotic resistance among enteric fever pathogens in a tertiary care centre. Natl J Lab Med. 2016;5:15-8 9. Hussain A, Satti L, Hanif F, Zehra NM, Nadeem S, Bangash TM, Peter A. Typhoidal Salmonella strains in Pakistan: An impending threat of extensively drug-resistant Salmonella Typhi. European Journal of Clinical Microbiology \& Infectious Diseases. 2019;38(11):2145-9

10. Marks F, Von Kalckreuth V, Aaby P, Adu-Sarkodie Y, El Tayeb MA, Ali M, et al. Incidence of invasive salmonella disease in sub-Saharan Africa: a multicentre population-based surveillance study. The Lancet Global Health. 2017;5(3):e31023

11. Andrews JR, Prajapati KG, Eypper E, Shrestha P, Shakya M, Pathak KR, et al. Evaluation of an electricity-free, culture-based approach for detecting typhoidal Salmonella bacteremia during enteric fever in a high burden, resource-limited setting. PLoS Negl Trop Dis. 2013;7(6):e2292

12. Capoor MR, Rawat D, Nair D, Hasan AS, Deb M, Aggarwal $\mathrm{P}$, et al. In vitro activity of azithromycin, newer quinolones and cephalosporins in ciprofloxacin-resistant Salmonella causing enteric fever. J Med Microbiol 2007;56:1490-4 